\title{
Asymmetric Dimethylarginine in Obese Youth: Relationship with 24-hour Ambulatory Blood Pressure
}

\author{
Polidori ${ }^{1}$, D’Adamo E ${ }^{2}$, Castorani $\mathrm{V}^{1}$, Mainieri $\mathrm{F}^{1}$, Chiarelli $\mathrm{F}^{1}$, Mohn $\mathrm{A}^{1}$ and Giannini $\mathrm{C}^{1 *}$ \\ ${ }^{1}$ Department of Pediatrics, University of Chieti, Chieti, Italy \\ ${ }^{2}$ Department of Neonatology, University of Chieti, Chieti, Italy
}

Corresponding author: Cosimo Giannini, Department of Pediatrics, University of Chieti, Via dei Vestini 5 I - 66100 Chieti, Italy

\section{ARTICLE INFO}

Received: 慧 October 10, 2020

Published: 慧 October 19, 2020

Citation: Polidori N, D’Adamo E, Castorani V, Mainieri F, Chiarelli F, Mohn A, Giannini C. Asymmetric Dimethylarginine in Obese Youth: Relationship with 24-hour Ambulatory Blood Pressure. Biomed J Sci \& Tech Res 31(2)-2020. BJSTR. MS.ID.005076.

Keywords: Obese adolescents; Blood pressure; Asymmetric dimethylarginine; Nitric Oxide; Oxidative Stress

\section{ABSTRACT}

Background: Asymmetric dimethylarginine (ADMA), a competitive inhibitor of nitric oxide synthase (NOS), is a marker for cardiovascular disease, a relevant issue in obesity. The aims of the present study were to evaluate ADMA, Nitric Oxide (NO) concentrations and oxidative stress (PGF- $2 \alpha$ ) in obese adolescents and to assess their potential association with twenty-four hour (24-h) ambulatory blood pressure (ABP).

Methods: A group of 117 obese adolescents were recruited and compared with 83 normal-weight age and sex matched peers. In all subjects, fasting blood samples were obtained for the evaluation of ADMA, NO, insulin, blood glucose and, urine samples to measure urinary PGF- $2 \alpha$. In addition, a $2 \mathrm{~h}$ oral glucose tolerance test was performed in all obese subjects. Blood pressure was evaluated by 24-h ABP monitoring.

Results: ADMA and urinary PGF- $2 \alpha(\mathrm{P}=0.0009$ and $\mathrm{P}=0.001$, respectively) levels were significantly increased while NO reduced $(\mathrm{P}=0.0005)$ in obese adolescents compared to controls. 24-h systolic and diastolic blood pressure (SBP and DBP) as well as daytime and night-time SBP and DBP were significantly higher in obese compared to controls (all $\mathrm{P}<0.05$ ). In a multiple stepwise linear regression analysis, the 24 -h blood pressure measurements were significantly associated to ADMA concentrations. After dividing the obese group by tertiles of ADMA, SBP and DBP significantly increased across tertiles. In addition to identify predictors of ADMA concentrations, a stepwise multiple regression was performed showing that oxidative stress and BMI-SDS were the most relevant factors affecting.

Conclusions: In obese youth, ADMA levels are increased and associated with impaired blood pressure. ADMA system might represent a novel potential target for hypertension in obese youth.

\section{Introduction}

Obesity represents one of the most important risk factors contributing to the overall burden of diseases worldwide [1,2]. Increased adiposity during childhood induces chronic metabolic and inflammatory alterations which are directly related to endothelial dysfunction and to the risk of cardiovascular diseases in adulthood [3]. Endothelial dysfunction is characterized by impaired nitric oxide (NO)-mediated vascular response and is associated with the development of cardiovascular complications in obese subjects
[4]. NO is an endothelium derived vasoactive mediator involved in several regulatory mechanisms within the cardiovascular system [5]. NO is formed from the amino acid precursor L-arginine by the enzyme NO-synthetase (NOS), whose activity is, in turn, negatively influenced by asymmetric dimethylarginine (ADMA), an arginine analogue. Increased ADMA concentrations have been reported in several diseases, including chronic renal failure, hyperhomocysteinemia, diabetes mellitus, hypercholesterolemia, hypertriglyceridemia, hypertension, pre-eclampsia, stroke, 
peripheral vascular diseases, congestive heart failure and acute coronary events [6]. NO release and/or bioavailability are also reduced in the context of insulin resistance and type 2 diabetes [6].

In a previous study, increased plasma ADMA levels were found in children with hypertension $[7,8]$. As well modifications in insulin resistance and ADMA concentrations seem to be implicated in changes of blood pressure moving from the pre-pubertal to the pubertal period in obese children. However, in this previous study no controls were available in order to characterized differences between obese and healthy normal-weight peers. In addition, no data on NO concentration and oxidative stress indexes were investigated. Therefore, the present study aimed to evaluate ADMA, NO and oxidative stress in well characterized group of only adolescents with obesity compared to age and sex matched healthy peers. In addition, we explored whether these markers might play a role in the regulation of blood pressure, as assessed by 24-hour ambulatory blood pressure (ABP) monitoring.

\section{Methods}

\section{Study Population}

We recruited 117 Caucasian obese adolescents (age $( \pm$ SD): 12.1 \pm 3.1 years; range 10.1-18.0 years) who had been referred to the Obesity Clinic of the Department of Pediatrics, University of Chieti, Italy. All subjects were defined obese on the basis of body mass index (BMI) >+2 SD for age and sex, using the Italian growth charts [9]. A detailed medical and family history was obtained for all subjects. None was taking any medication known to affect body composition or blood pressure and none were affected by any medical illnesses. A complete physical examination was performed, including anthropometric parameters and staging of puberty according to Tanner criteria. Fasting blood and overnight urine samples were obtained. In obese adolescents, a standard oral glucose tolerance test (OGTT) was performed in order to evaluate glucose tolerance. As a control group, we recruited 83 normal weight (BMI between -2 and +2 SD) healthy children comparable for age (age $12.7 \pm 3.4$ years; range 10.2-17.7 years), gender and pubertal stage, who had been admitted to the Department of Paediatrics of the University of Chieti for minor diseases. Control subjects were evaluated at least 8 weeks after discharge for any minor disease. The local Ethics Committee of the University of Chieti approved the study. Informed consent was obtained by parents and assent from the children.

\section{Anthropometric Measurements}

Height was measured in triplicate with a wall mounted stadiometer to the nearest $0.01 \mathrm{~m}$ and body weight was measured with a digital scale to the nearest $0.1 \mathrm{~kg}$. BMI was expressed as $\mathrm{kg} /$ $\mathrm{m}^{2}$ and the standard deviation score for BMI (BMI-SDS) for age and sex was calculated for each subjects according to Italian growth chart [9]. In all subjects, pubertal stage was defined on the basis of breast development in girls and genital development in boys, according to Tanner's criteria.

\section{Biochemical Procedures}

Fasting plasma glucose and insulin levels were measured. Multiple aliquots of fasting blood samples were collected and stored at $-80{ }^{\circ} \mathrm{C}$ for later assessment of ADMA and serum NO. All subjects performed an overnight urine collection before blood sampling. Urine samples were added with the antioxidant 4-hydroxy-tempo (Sigma Chemical Co, St Louis; Mo) and multiple aliquot samples were stored at $-80^{\circ} \mathrm{C}$ until analysis of urinary isoprostanes (PGF$2 \alpha)$.

\section{Oral Glucose Tolerance Test and Insulin Resistance Indices}

In all obese subjects, a standard 2-hour OGTT was performed according to standard procedures, as previously described [10]. HOMA-IR [(Fasting Insulin*Fasting Glucose)/22.5] was calculated as insulin resistance index. Plasma glucose level was determined by using the glucose oxidase method and plasma insulin was measured with two-site immunoenzymometric assay (AIA-PACK IRI; Tosoh, Tokyo, Japan). The limit of detection was $0.5 \mu \mathrm{U} / \mathrm{mL}$ with intra- and interassay coefficients of variation $<7 \%$ for quality control.

\section{ADMA}

Plasma ADMA concentration was determined by a validated ELISA kit (DLD Diagnostika, Hamburg, Germany) which has previously been described and validated in detail [11]. In brief, cross-reactivity was $1.2 \%$ for symmetric dimethylarginine (SDMA) and $<0.02 \%$ for L-arginine. The limit of detection was $0.05 \mu \mathrm{mol} / \mathrm{l}$. The intra- and the inter-assay variation was 5.7 and 8.6 (CV \%), respectively. There is a good correlation of the values measured by this ELISA and LC-tandem MS $(n=29 ; \mathrm{R}=0.984 ; \mathrm{P}<0.0001)$. Reference ranges for ADMA have been established by this technique recently [12].

\section{Nitric Oxide}

Serum NO was measured in triplicate using a classical twostep assay. In detail, NO was defined by the sum of its metabolites nitrite and nitrate, defined following the conversion of nitrate to nitrite using a commercially available kit based on the Griess reaction (Total Nitric Oxide Assay kit; Assay Design, Ann Arbor, MI) [13]. The consumption of foods containing nitrates (i.e., spinach, beet, cabbage, cauliflower, beetroot, lettuce, among others) was discouraged for the $48 \mathrm{~h}$ preceding the measurements and evaluated by a two days dietary diary to avoid any dietary influence on the results of the NO assay.

\section{Urinary Isoprostanes}

Urinary samples were added with the antioxidant 4-hydroxytempo (Sigma Chemical Co, St Louis; Mo) and multiple aliquot samples were stored at $-80^{\circ} \mathrm{C}$ until the analysis was performed. PGF- $2 \alpha$ levels were evaluated in triplicate by an immuno-enzymatic method (8-iso-Prostaglandin F2 $\alpha$ Enzyme Immunoassay kit; Assay 
Design) [14].

\section{Ambulatory Blood Pressure Monitoring (ABPM)}

ABPM was performed with a portable non-invasive oscillometric recorder (SpaceLabs model 90207; Redmond, Washington, USA). The validity of this monitor has been previously confirmed $[15,16]$. The left arm was used in all children, according to the cuffs designed, and three different cuff sizes were used $(10 \times 13 \mathrm{~cm}, 13 \mathrm{x}$ 24 and $24 \times 32 \mathrm{~cm}$ ), selected on the basis of the arm circumference of each child. ABP was performed during a normal day with typical activities but children were asked to avoid vigorous exercises and to keep their arm relaxed during each day-time inflation. Adolescents and parents were asked to record in a diary the time they went to bed and the time they awoke, as well as exercise periods and the quality of the sleep. At the beginning of the evaluation period, the accuracy and precision of the monitor was confirmed by simultaneous measurements with a mercury sphygmomanometer.

Blood pressure readings were obtained at 20-min intervals during daytime and at 30-min intervals during night-time. At the end of the ABP, the monitor was downloaded to a personal computer for the analysis of the measurements. All readings taken during 24-h were used to calculated mean 24-h systolic blood pressure (SBP) and diastolic blood pressure (DBP). All readings taken during 24-h were used to calculate mean 24-h SBP, DBP and HR, whereas mean daytime and night-time SBP, DBP and HR were calculated based on the awake and asleep periods, calculated from diary times [17]. Recordings were automatically rejected if systolic blood pressure was $>240$ or $<70 \mathrm{mmHg}$ or if diastolic blood pressure was $>150$ or $<40 \mathrm{mmHg}$ or if heart rate $>200$ or $<20$ beats / min. All ambulatory blood pressure parameters were converted in SDS using the normative values published by Wuhl, et al. [18].

\section{Statistical Analysis}

Statistical analyses were performed with SPSS (22 for Windows, SPSS Inc., Chicago, IL). All data were expressed as mean \pm standard deviation or $95 \%$ CI. For all analyses, a P-value $<0.05$ was considered statistically significant. The distribution of continuous variables was tested for skewness and kurtosis and those variables not normally distributed were logarithmically transformed. Differences in sex distribution were analyzed by Fisher's exact test while dichotomic variables were analyzed by Pearson chi-square tests. Differences for continuous variables between obese and control adolescents were assessed by performing the analysis of covariance (ANCOVA), adjusting for age and sex. To identify factors affecting blood pressure, a stepwise multiple regression was performed using 24h SBP-SDS and DBP-SDS values as dependent variable. The parameters included in the linear regression model as independent variables were age, gender, BMI-SDS, HOMA-IR, ADMA and PGF- $2 \alpha$.

In order to further assess the effects of ADMA concentration on ABP, obese subjects were divided according to tertiles of ADMA. Analysis of variance with Tukey's post-hoc testing was performed.
Adjustment for potential confounding factors was performed using analysis of covariance adjusting for age, gender, BMI-SDS. In addition to identify predictors of ADMA concentrations, a stepwise multiple regression was performed using ADMA concentrations as dependent variable. The parameters included in the linear regression model as independent variables were age, gender, BMISDS, 2-hr glucose, HOMA-IR and PGF-2 $\alpha$. Variables of interest were first tested singularly and then all together in a single model in order to test the best predictors of ADMA concentrations.

\section{Results}

\section{Obese and Control Subjects}

Obese and control subjects were similar for age, sex and pubertal stage. As expected, BMI and BMI-SDS were significantly higher in obese children than in controls (both $\mathrm{P}<0.001$ ); while no difference was documented for height (Table 1). Obese children also showed higher levels of fa sting insulin, fasting glucose and HOMA-IR values (all $\mathrm{P}<0.001$ ) than controls. None of the obese adolescents had impaired fasting glucose, impaired glucose tolerance (IGT) or type 2 diabetes (Table 1 ).

Table 1: Main anthropometric and metabolic parameters of obese and control subjects.

\begin{tabular}{|c|c|c|c|}
\hline & Obese & Controls & $\mathbf{P}$ \\
\hline \multicolumn{4}{|l|}{ Anthropometric parameters } \\
\hline Number & 117 & 83 & \\
\hline $\operatorname{Sex}(F / M)$ & $56 / 61$ & $43 / 40$ & \\
\hline Age (years) & $12.1 \pm 3.1$ & $12.7 \pm 3.4$ & 0.09 \\
\hline Height-SDS & $0.3 \pm 1.0$ & $0.2 \pm 1.0$ & 0.46 \\
\hline BMI (kg/m2) & $31.0 \pm 6.2$ & $18.84 \pm 2.72$ & $<0.001$ \\
\hline BMI-SDS & $2.4 \pm 0.6$ & $-0.2 \pm 0.7$ & $<0.001$ \\
\hline \multicolumn{4}{|l|}{ Metabolic parameters } \\
\hline Fasting Glucose (mg/dl) & $91 \pm 9$ & $81 \pm 8$ & $<0.001$ \\
\hline 2-h Glucose (mg/dl) & $109 \pm 17$ & & \\
\hline Fasting Insulin $(\mu \mathrm{U} / \mathrm{mL})$ & $17.8 \pm 13.4$ & $9.5 \pm 4.7$ & $<0.001$ \\
\hline HOMA-IR & $3.87 \pm 3.09$ & $1.88 \pm 0.92$ & $<0.001$ \\
\hline \multicolumn{4}{|l|}{ 24-h ABPM } \\
\hline SBP (mmHg) & $117 \pm 11$ & $111 \pm 8$ & 0.003 \\
\hline SBP-SDS (mmHg) & $0.83 \pm 1.13$ & $0.02 \pm 1.14$ & $<0.001$ \\
\hline DBP (mmHg) & $67 \pm 6$ & $64 \pm 6$ & 0.001 \\
\hline DBP-SDS (mmHg) & $0.04 \pm 1.01$ & $-0.53 \pm 1.01$ & $<0.001$ \\
\hline HR (bpm) & $82 \pm 7$ & $80 \pm 9$ & 0.17 \\
\hline \multicolumn{4}{|l|}{ Day-Time ABPM } \\
\hline SBP (mmHg) & $119 \pm 9$ & $115 \pm 7$ & 0.004 \\
\hline SBP-SDS (mmHg) & $0.55 \pm 0.94$ & $0.16 \pm 1.01$ & $<0.001$ \\
\hline DBP (mmHg) & $69 \pm 6$ & $66 \pm 5$ & $<0.001$ \\
\hline DBP-SDS (mmHg) & $-0.04 \pm 1.10$ & $-0.89 \pm 0.92$ & 0.001 \\
\hline HR (bpm) & $84 \pm 9$ & $84 \pm 8$ & 0.87 \\
\hline \multicolumn{4}{|l|}{ Night-Time ABPM } \\
\hline SBP (mmHg) & $110 \pm 11$ & $106 \pm 11$ & 0.02 \\
\hline
\end{tabular}




\begin{tabular}{|c|c|c|c|}
\hline SBP-SDS (mmHg) & $0.65 \pm 1.16$ & $0.21 \pm 1.22$ & 0.01 \\
\hline DBP (mmHg) & $60 \pm 6$ & $58 \pm 7$ & 0.03 \\
\hline DBP-SDS (mmHg) & $-0.02 \pm 1.06$ & $-0.30 \pm 1.12$ & 0.04 \\
\hline HR (bpm) & $77 \pm 10$ & $75 \pm 12$ & 0.09 \\
\hline
\end{tabular}

Data are mean $\pm S D$

$\mathrm{M}=$ Male; $\mathrm{F}=$ Female; $\mathrm{SDS}=$ Standard Deviation Score; $\mathrm{BMI}=$ Body Mass Index; HOMA-IR= Homeostasis Model Assessment of Insulin Resistance; SBP=Systolic Blood Pressure; DBP= Diastolic Blood Pressure; HR= Heart Rate; ABPM= Ambulatory Blood Pressure Measurements

\section{ABP (Table 1)}

Compared to controls, both SBP and DBP values for 24-h, dayand night-time were significantly increased in obese adolescents (all $\mathrm{P}<0.05$ ). Similarly, both SBP-SDS and DBP-SDS values for 24-h, day- and night-time were significantly increased in obese adolescents (all $\mathrm{P}<0.05$ ) (Table 1). No difference was documented in terms of HR. Out of 117 obese subjects, 16 adolescents presented hypertension as defined by values of systolic or diastolic or both $\geq 95$ th percentile (10 with a mean 24 -h SBP value $\geq 95$ th percentile, 2 with a mean 24 -h DBP value $\geq 95$ th percentile; 4 with both mean 24-h SBP and DBP values $\geq 95$ th percentile).

\section{ADMA, NO and Oxidative Stress (Figure 1)}

As shown in Figure 1, in obese adolescents ADMA levels were significantly higher compared to controls $(0.65 \pm 0.19$ vs $0.53 \pm 0.14$ $\mu \mathrm{mol} / \mathrm{L}, \quad \mathrm{P}=0.0009$ ), whereas serum NO levels were lower (50.26 \pm 18.97 vs $87.10 \pm 43.51 \mathrm{mmol} / \mathrm{L}, \mathrm{P}=0.0005$ ). PGF- $2 \alpha$ levels were higher in obese children compared to controls $(20.62 \pm 13.42$ vs $14.18 \pm 9.18 \mathrm{ng} / \mathrm{ml}, \mathrm{P}=0.001$ ) (Figure 1).
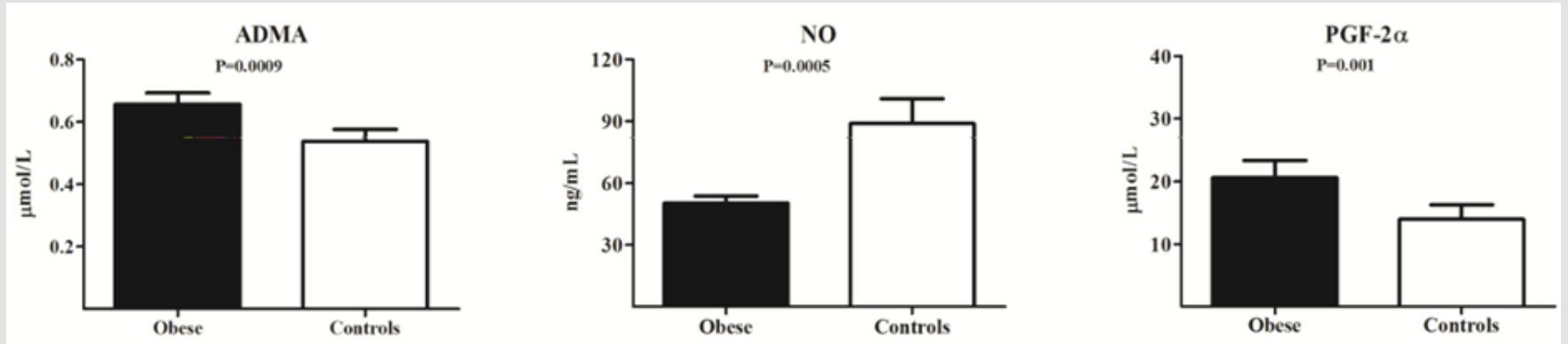

Figure 1: Asymmetric dimethylarginine (ADMA), Nitric Oxide (NO) and oxidative stress (PGF-2a) levels in Obese adolescents and controls

\section{Factors Affecting Blood Pressure in Obese Subjects}

In order to evaluate the potential influence of the most relevant variables of interest on blood pressure, a multiple stepwise regression analysis was performed using the 24h SBP-SDS and DBP-SDS as dependent variables. Of note, adjusting for age and gender, ADMA concentrations were significantly associated to both 24h SBP-SDS and DBP-SDS ( $\mathrm{P}=0.001, \beta=0.41$ and $\mathrm{P}=0.002, \beta=0.32$ ) while BMI-SDS, HOMA-IR and PGF- $2 \alpha$ were excluded by the model (all $\mathrm{P}>0.05$, adjusted for age and gender).

\section{Obese Subjects Divided According to ADMA Tertile (Figure 2) and Multiple Stepwise Linear Regression Analysis (Table 2)}

In order to evaluate the potential influence of ADMA concentrations on blood pressure, obese adolescents were categorized according to tertiles of ADMA (first tertile: <0.58; second tertile: 0.58 and 0.75 ; third tertile $>0.75$ ) (Figure 2). As shown in Figure 2, 24-h SBP-SDS and 24-h DBP-SDS progressively increased across ADMA tertiles (all $\mathrm{P}$ for trend $\mathrm{P} \leq 0.002$ ) (Figure 2). Similarly, day- and night- both SBP-SDS and DBP-SDS, progressively increased across ADMA tertiles (all $\mathrm{P}$ for trend $\mathrm{P} \leq 0.002$ ). As expected, all parameters used to evaluate glucose metabolism and insulin resistance as well as oxidative stress markers increased progressively according tertiles of ADMA (data not shown).

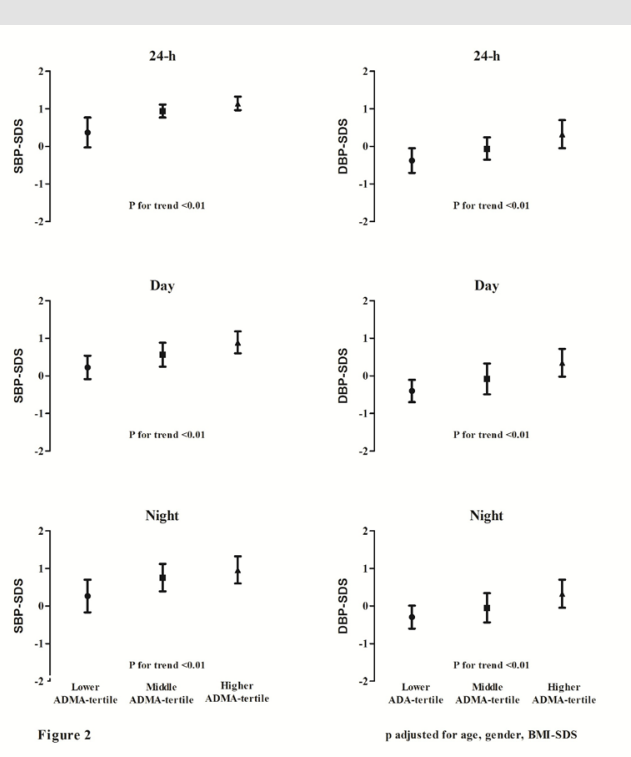

Figure 2: Distribution of 24-h-, day- and night- SBP-SDS and DBP-SDS in obese subjects divided according to ADMA tertiles (all $\mathrm{P}$ for trend $\mathrm{P} \leq 0.001$ ). 
In order to evaluate factors affecting ADMA levels a multiple stepwise linear regression analysis was performed. As shown in Table 2, when the single variables of interest were included in the model, BMI-SDS, 2-h glucose, HOMA-IR and oxidative stress showed a significant and direct association with ADMA levels (Table 2). Of note, when these variables were included all together in the model, oxidative stress and BMI-SDS showed the strongest association with ADMA concentrations (Table 2).

Table 2: Multiple stepwise linear regression analysis evaluating factors influencing ADMA levels (dependent variables) in obese adolescents.

\begin{tabular}{|c|c|c|c|c|c|c|c|c|c|c|}
\hline Independent variables & $\boldsymbol{\beta}$ & $\mathbf{P}$ & $\boldsymbol{\beta}$ & $\mathbf{P}$ & $\boldsymbol{\beta}$ & $\mathbf{P}$ & $\boldsymbol{\beta}$ & $\mathbf{P}$ & $\boldsymbol{\beta}$ & $\mathbf{P}$ \\
\hline BMI-SDS & 0.29 & 0.001 & & & & & & & 0.22 & 0.03 \\
\hline 2-h Glucose & & & 0.3 & 0.002 & & & & & 0.17 & 0.1 \\
\hline HOMA-IR & & & & & 0.22 & 0.01 & & 0.14 & 0.15 \\
\hline PGF-2 $\alpha$ & & & & & & & 0.38 & 0.0005 & 0.33 & 0.002 \\
\hline
\end{tabular}

Adjusted for age and gender

BMI-SDS= Body Mass Index-Standard Deviation Score; PGF-2a= Urinary Isoprostanes

\section{Discussion}

In this study, we showed that obese adolescents had higher ADMA and lower NO concentrations, compared to healthy matched peers. BMI, 2-h glucose and oxidative stress were independently determinants of ADMA levels. Notably, in this group of subjects impaired ADMA concentrations appeared to be associated with increased systolic and diastolic blood pressure, as assessed by $\mathrm{ABP}$, suggesting a potential role of ADMA on blood pressure dysregulation. During the last decade, the epidemic increase in childhood obesity has determined a shift from secondary forms of hypertension, once predominant in this age group, to primary hypertension, previously considered to be rare in children [1921]. In fact, in obese children adiposity and insulin resistance have shown to play a major role in influencing blood pressure [22]. Release of ADMA into the plasma as well intracellular production of ADMA inhibits NOS, reducing the bioavailability of NO [4-6]. NO is an anti-atherogenic molecule, which acts as a vasodilatant, inhibits platelet aggregation, smooth muscle cell proliferation and expression of adhesion molecules [4-6]. Kielstein et al. [23] showed that systemic administration of ADMA reduces NO generation, renal perfusion, and sodium excretion in healthy humans [23]. In a previous study in hypertensive children, plasma levels of ADMA and of its stereoisomer, symmetric dimethylarginine, were increased and associated with office blood pressure measurements. In addition previous data have shown that changes in BP already occur moving from the pre-pubertal to the pubertal period in obese children, and modifications in insulin resistance and ADMA seem to be implicated in this early progression in BP. However, no data regarding NO concentrations were available and more importantly no controls and no data on glucose tolerance status were available.

In this study we found that ADMA concentrations were increased and associated with decreased NO values in obese adolescents compared to healthy matched controls. Of note these alterations in the physiological balance between ADMA/NO system appeared to be directly related to alterations in blood pressure regulation. In fact, a progressive increase in blood pressure values were found across tertiles of ADMA, and this trend persisted even after adjusting for potential confounding factors. Taken together, our results suggest a role of ADMA as a risk factor for abnormal blood pressure in obese adolescents, and a potential target for future intervention strategies. Several mechanisms might explain the finding of increased ADMA concentration in obese adolescents [25-27]. In vitro, in vascular smooth muscle and endothelial cells incubated in a hyperglycemic medium, as well as in streptozotocininduced diabetic rats, hyperglycemia has been shown to reduce dimethylarginine dimethylaminohydrolase (DDAH) activity, resulting in increased ADMA concentration [28, 29]. In addition, ADMA levels have been shown to be directly correlated with 2-h glucose [30].

Similarly, an acute increase of $33 \%, 30 \%$ and $45 \%$ of ADMA concentration after a 2-h OGTT has been reported in subjects with normal glycaemia, impaired glucose tolerance and type 2 diabetes, respectively [31]. In line with these findings, we found that 2-hour post-challenge glucose levels were associated with ADMA levels already in a state of normal glucose tollerance. It is thought that ADMA may also accumulate due to increased production, as a result of redox-sensitive activation of protein arginine N-methyltransferases (PRMTs) which form ADMA [32]. It is therefore thought that oxidative stress results in elevated levels of ADMA that inhibit NOS activity [33]. In non-diabetic subjects there is a significant correlation between plasma ADMA concentrations and insulin resistance [34, 35], and improvement in insulin sensitivity with rosiglitazone or weight loss results in a fall in ADMA $[34,35]$. In obese and elderly subjects, increased ADMA appears to be related to decreased insulin sensitivity [30]. Interestingly in our population oxidative stress was one of the main factors associated with ADMA levels.

The epidemic increase in childhood obesity has caused a shift from secondary forms of hypertension, once predominant in this age group, to primary hypertension, previously considered to be 
rare in children. In obese youth, systemic arterial hypertension represents a relevant cardiovascular risk factor. We showed that ADMA/NO system is impaired in obese youth compared with healthy peers and seems to be directly involved in blood pressure alteration in obese youth. Taken together, our results postulate a role of ADMA concentrations as a risk factor for high blood pressure in obese adolescents and a potential target for future interventional strategies. The strength of the current study stems from the assessment of blood pressure and the complete ADMA/ NO/oxidative stress axis in a large group of only adolescent obese subjects compared to healthy normal-weight age, gender and pubertal state matched peers. In addition blood pressure was evaluated by using $\mathrm{ABP}$, a simple, non-invasive method of measuring blood pressure, which permits the observation of blood pressure throughout day and night in a nonmedical environment and quantification of circadian blood pressure variability and to minimizing the effect of potential confounders, such as the white coat effect [17, 36-38]. The major limitation of our study may be represented by its cross-sectional design, therefore requiring confirmation in future longitudinal studies.

In conclusion, obese youths present increased ADMA and decreased NO levels compared with healthy matched peers, and these markers are associated with impaired blood pressure regulation. Adiposity and oxidative stress represent the major factors influencing ADMA levels in this age group.

\section{References}

1. Güngör NK (2014) Overweight and obesity in children and adolescents. J Clin Res Pediatr Endocrinol 6(3): 129-43.

2. Lee EY, Yoon KH (2018) Epidemic obesity in children and adolescents: risk factors and prevention. Front Med 12: 658-666.

3. Weihrauch-Blüher S, Schwarz P, Klusmann JH (2019) Childhood obesity: increased risk for cardiometabolic disease and cancer in adulthood. Metabolism 92: 147-152.

4. Schulz E, Gori T, Munzel T (2010) Oxidative stress and endothelial dysfunction in hypertension. Hypertens Res 34(6): 665-673.

5. Bruckdorfer R (2005) The basics about nitric oxide. Mol Aspects Med 26(1-2): 3-31.

6. Boger RH (2006) Asymmetric dimethylarginine (ADMA): a novel risk marker in cardiovascular medicine and beyond. Ann Med 38(2): 126136.

7. de Giorgis T, Marcovecchio ML, Giannini C, Chiavaroli V, Chiarelli F, et al. (2016) Blood pressure from childhood to adolescence in obese youths in relation to insulin resistance and asymmetric dimethylarginine. Endocrinol Invest 39: 169-76.

8. Goonasekera CD, Rees DD, Woolard P, Frend A, Shah V, et al. (1997) Nitric oxide synthase inhibitors and hypertension in children and adolescents. J Hypertens 15(8): 901-909.

9. Cacciari E, Milani S, Balsamo A, Spada E, Cavallo L, et al. (2006) Italian cross-sectional growth charts for height, weight and BMI (2 to $20 \mathrm{yr}$ ). J Endocrinol Invest 29(7): 581-593.

10. Jiang B, Tang G, Cao K, Wu L, Wang R (2010) Molecular mechanism for H(2)S-induced activation of K(ATP) channels. Antioxid Redox Signal 12: 1167-1178.
11. Schulze F, Wesemann R, Schwedhelm E, Sydow K, Albsmeier J, et al. (2004) Determination of asymmetric dimethylarginine (ADMA) using a novel ELISA assay. Clin Chem Lab Med 42(12): 1377-1383.

12. Schulze F, Maas R, Freese R, Schwedhelm E, Silberhorn E, et al. (2005) Determination of a reference value for $N(G), N(G)$-dimethyl-L-arginine in 500 subjects. Eur J Clin Invest 35(10): 622-626.

13. Wennmalm A, Benthin G, Edlund A, Jungersten L, Jensen NK, et al. (1993) Metabolism and excretion of nitric oxide in humans. An experimental and clinical study. Circ Res 73(6): 1121-1127.

14. Roberts LJ, Morrow JD (2000) Measurement of F(2)-isoprostanes as an index of oxidative stress in vivo. Free Radic Biol Med 28(4): 505-513.

15. O’Brien E, Mee F, Atkins N, O'Malley K (1991) Accuracy of the SpaceLabs 90207 determined by the British Hypertension Society protocol. J Hypertens 9(6): 573-574.

16. Reichert H, Lindinger A, Frey O, J Mortzeck, J Kiefer, et al. (1995) Ambulatory blood pressure monitoring in healthy schoolchildren. Pediatr Nephrol 9: 282-286.

17. Soergel M, Kirschstein M, Busch C (1997) Oscillometric twenty-four-hour ambulatory blood pressure values in healthy children and adolescents: a multicenter trial including 1141 subjects. J Pediatr 130: 178-184.

18. Wuhl E, Witte K, Soergel M, Mehls O, Schaefer F, et al. (2002) Distribution of 24-h ambulatory blood pressure in children: normalized reference values and role of body dimensions. J Hypertens 20(10): 1995-2007.

19. Caprio S, Santoro N, Weiss R (2020) Childhood obesity and the associated rise in cardiometabolic complications. Nat Metab 2: 223-232.

20. Wühl E (2019) Hypertension in childhood obesity. Acta Paediatr 108: 37-43.

21. Muntner P, He J, Cutler JA, Wildman RP, Whelton PK (2004) Trends in blood pressure among children and adolescents. JAMA 291(17): 2107 2113.

22. Marcovecchio ML, Patricelli L, Zito M, Capanna R, Ciampani M, et al. (2006) Ambulatory blood pressure monitoring in obese children: role of insulin resistance. J Hypertens 24(12): 2431-2436.

23. Kielstein JT, Impraim B, Simmel S, Bode-Böger SM, Tsikas D, et al. (2004) Cardiovascular effects of systemic nitric oxide synthase inhibition with asymmetrical dimethylarginine in humans. Circulation 109(2): 172-177.

24. White WB (2000) Ambulatory blood pressure monitoring: dippers compared with non-dippers. Blood Press Monit 5(Suppl 1): S17-23.

25. Palm F, Onozato ML, Luo Z, Wilcox CS (2007) Dimethylarginine dimethylaminohydrolase (DDAH): expression, regulation, and function in the cardiovascular and renal systems. Am J Physiol Heart Circ Physiol 293(6): H3227-3245.

26. Liu X, Xu X, Shang R, Chen Y (2018) Asymmetric dimethylarginine (ADMA) as an important risk factor for the increased cardiovascular diseases and heart failure in chronic kidney disease. Nitric Oxide 78: 113-120.

27. Achan V, Broadhead M, Malaki M, Whitley G, Leiper J, et al. (2003) Asymmetric dimethylarginine causes hypertension and cardiac dysfunction in humans and is actively metabolized by dimethylarginine dimethylaminohydrolase. Arterioscler Thromb Vasc Biol 23(8): 14551459 .

28. Lin KY, Ito A, Asagami T, Adimoolam S, Kimoto M, et al. (2002) Impaired nitric oxide synthase pathway in diabetes mellitus: role of asymmetric dimethylarginine and dimethylarginine dimethylaminohydrolase. Circulation 106(8): 987-992.

29. Lu CW, Guo Z, Feng M, Wu ZZ, He ZM, et al. (2010) Ex vivo gene transferring of human dimethylarginine dimethylaminohydrolase- 2 improved endothelial dysfunction in diabetic rat aortas and high glucose-treated endothelial cells. Atherosclerosis 209(1): 66-73. 
30. Marliss EB, Chevalier S, Gougeon R, JA Morais, M Lamarche, et al. (2006) Elevations of plasma methylarginines in obesity and ageing are related to insulin sensitivity and rates of protein turnover. Diabetologia 49: 351359.

31. Konukoglu D, Firtina S, Serin O (2008) The relationship between plasma asymmetrical dimethyl-L-arginine and inflammation and adhesion molecule levels in subjects with normal, impaired, and diabetic glucose tolerance. Metabolism 57(1): 110-115.

32. Boger RH, Sydow K, Borlak J, Thum T, Lenzen H, et al. (2000) LDL cholesterol upregulates synthesis of asymmetrical dimethylarginine in human endothelial cells: involvement of S-adenosylmethioninedependent methyltransferases. Circ Res 87(2): 99-105.

33. Blackwell S (2010) The biochemistry, measurement and current clinical significance of asymmetric dimethylarginine. Ann Clin Biochem 47(1): 17-28.

34. Stuhlinger MC, Abbasi F, Chu JW, Lamendola C, Tracey L McLaughlin, et al. (2002) Relationship between insulin resistance and an endogenous nitric oxide synthase inhibitor. JAMA 287(11): 1420-1426.

ISSN: 2574-1241

DOI: $10.26717 /$ BJSTR.2020.31.005076

Giannini C. Biomed J Sci \& Tech Res

(C) This work is licensed under Creative

Submission Link: https://biomedres.us/submit-manuscript.php
35. McLaughlin T, Stuhlinger M, Lamendola C, Abbasi F, Bialek J, et al. (2006) Plasma asymmetric dimethylarginine concentrations are elevated in obese insulin-resistant women and fall with weight loss. J Clin Endocrinol Metab 91(5): 1896-1900.

36. Lurbe E, Torro I, Alvarez V, Nawrot T, Paya R, et al. (2005) Prevalence, persistence, and clinical significance of masked hypertension in youth. Hypertension 45(4): 493-498.

37. Koch VH, Colli A, Saito MI, Furusawa EA, Ignes E, et al. (2000) Comparison between casual blood pressure and ambulatory blood pressure monitoring parameters in healthy and hypertensive adolescents. Blood Press Monit 5(5-6): 281-289.

38. Wuhl E, Witte K, Soergel M, Mehls O, Schaefer F (2002) Distribution of 24-h ambulatory blood pressure in children: normalized reference values and role of body dimensions. J Hypertens 20(10): 1995-2007.

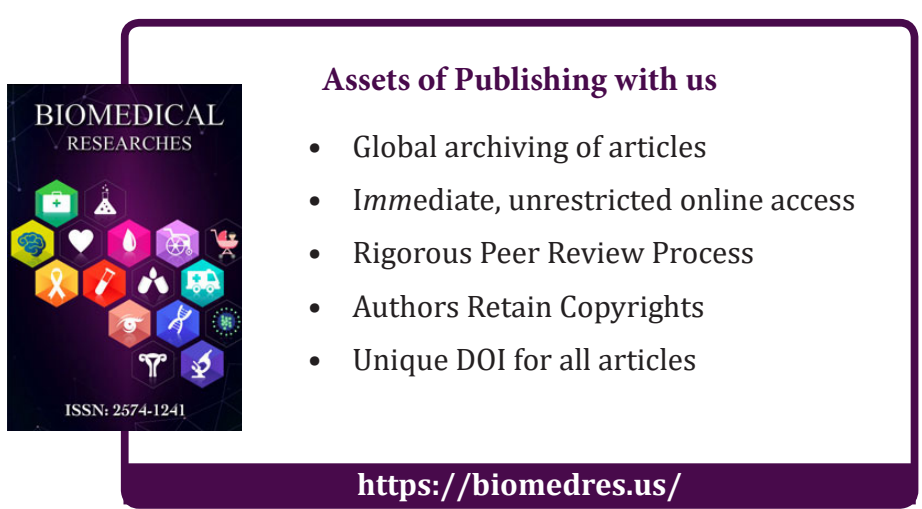

